\title{
Surgical wound dehiscence complicated by methicillin- resistant Staphylococcus aureus infection in a diabetic patient with femorotibial vascular bypass occlusion
}

\author{
Authors: Enrico M Zardi, ${ }^{\mathrm{A}}$ Nunzio Montelione, ${ }^{\mathrm{B}}$ Rossella C Vigliotti, ${ }^{\mathrm{C}}$ Camilla Chello, ${ }^{\mathrm{D}}$ Domenico M Zardi, ${ }^{\mathrm{C}}$ \\ Francesco Spinelli ${ }^{F}$ and Francesco Stilo $^{G}$
}

\begin{abstract}
Diabetic patients with critical limb ischaemia may be affected by severe wound and skin ulcer infections. We report a case of a patient with bilateral femorotibial occlusion and methicillinresistant Staphylococcus aureus infection. The patient was treated with femoroperoneal vascular bypass, debridement of wound dehiscence and targeted antimicrobial therapy for symptom resolution and healing of the wound.
\end{abstract}

KEYWORDS: Diabetes, infection, skin ulcer, therapy, vascular bypass

\section{Introduction}

Foot infections are common disabling problems affecting the vascular management of diabetic patients resulting in a high rate of amputation and mortality depending on foot-related risk characteristics. ${ }^{1,2}$ Prognosis is related to efficacious prevention and early treatment, being worse based on the severity of clinical presentation (ulcer, gangrene and sepsis).

We describe a diabetic patient with critical limb ischaemia due to femorotibial vascular bypass occlusion and wound infection.

\section{Case presentation}

A 67-year-old man with critical limb ischaemia presented to the hospital; he had type 2 diabetes, chronic renal failure, chronic bronchitis complicated by some episodes of pneumonia in previous years and myocardial infarction (in 2010 and 2015) treated with coronary revascularisation.

In November 2017 he developed a bilateral calcaneal skin ulcer; in April 2018, he underwent bilateral femoral profundoplasty

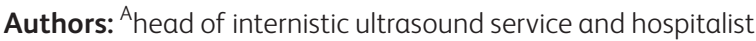
consultant, Campus Bio-Medico University, Rome, Italy; ${ }^{\text {Bvascular }}$ surgery specialist, Campus Bio-Medico University, Rome, Italy; ${ }^{C}$ vascular surgery specialist, Campus Bio-Medico University, Rome, Italy; ' dermatology specialist, University La Sapienza, Rome, Italy; Einterventional cardiology specialist, University La Sapienza, Rome, Italy; ${ }^{F}$ head of division of vascular surgery, Campus Bio-Medico University, Rome, Italy; ${ }^{G}$ professor of vascular surgery, Campus BioMedico University, Rome, Italy combined with endarterectomy of the superficial femoral artery. A month later he underwent the calcaneal skin ulcers debridement.

The wound specimen for bacterial culture was positive for Staphylococcus haemolyticus, Corynebacterium aurimucosum and Corynebacterium simulans, he was treated with intravenous teicoplanin at $400 \mathrm{mg}$ once per day and intravenous meropenem at 500 mg every 8 hours. In June and July, he was admitted to our hospital for rest pain and persistence of bilateral calcaneal ulcers; he was treated with left femorotibial posterior bypass and right femorotibial anterior bypass, using the greater saphenous vein. After an additional 3 months, he was newly admitted in our hospital for wound dehiscence debridement. The collection of culture samples resulted in isolation of Pseudomonas aeruginosa and Enterobacter cloacae; these were intravenously treated, according to antibiogram, with $500 \mathrm{mg}$ of ciprofloxacin every 12 hours and gentamicin at a dosage of $3 \mathrm{mg} / \mathrm{kg} /$ day, for 10 days. Finally, in January 2019 he was treated for critical limb ischaemia of grade 3 (Wound, Ischemia and foot Infection classification). ${ }^{3}$ On admission, he presented wound dehiscence with mixed infections (Fig 1). Laboratory analysis showed C-reactive protein of $15 \mathrm{mg} / \mathrm{dL}$, white blood cells count of 12,150 cells $/ \mu \mathrm{L}$, neutrophils count of 10,200 cells $/ \mu \mathrm{L}(84 \%)$, red blood cells count of $4,500,000$ cells $/ \mu \mathrm{L}$, haemoglobin of $8.9 \mathrm{~g} / \mathrm{dL}$, platelets count of $450,000 / \mu \mathrm{L}$, fibrinogen of $757 \mathrm{mg} / \mathrm{dL}(<400)$ and creatinine of $0.99 \mathrm{mg} / \mathrm{dL}$.

The ankle-brachial index was $<1$. Computed tomography showed occlusion of both femorotibial vascular bypass (Fig 2). Administration of metformin was started, beginning with a dosage of $500 \mathrm{mg}$ once per day during lunch. He underwent bilateral femoroperoneal bypass through lesser saphenous vein and then the debridement of both wounds. The specimens for bacterial culture were positive for methicillin-resistant Staphylococcus aureus (MRSA; Fig 3). In accordance with antibiogram sensitivity and guidelines for MRSA treatment, intravenous daptomycin at a dosage of $6 \mathrm{mg} / \mathrm{kg} /$ day and oral rifampicin at a total dosage of $600 \mathrm{mg} /$ day were started and continued for 20 days. ${ }^{4}$ At home, he continued intramuscular teicoplanin at 400 mg every 12 hours and oral rifampicin at a dosage of $600 \mathrm{mg} /$ day for 7 days. His C-reactive protein diminished to $2.57 \mathrm{mg} / \mathrm{dL}$. Glycaemic control (glycated haemoglobin of $6 \%$ ) was obtained increasing the administration of metformin at $500 \mathrm{mg}$ three times per day. Healing of the limb wound and regression of rest pain was obtained (Fig 4). At 6 months follow-up, colour Doppler sonography showed patency 


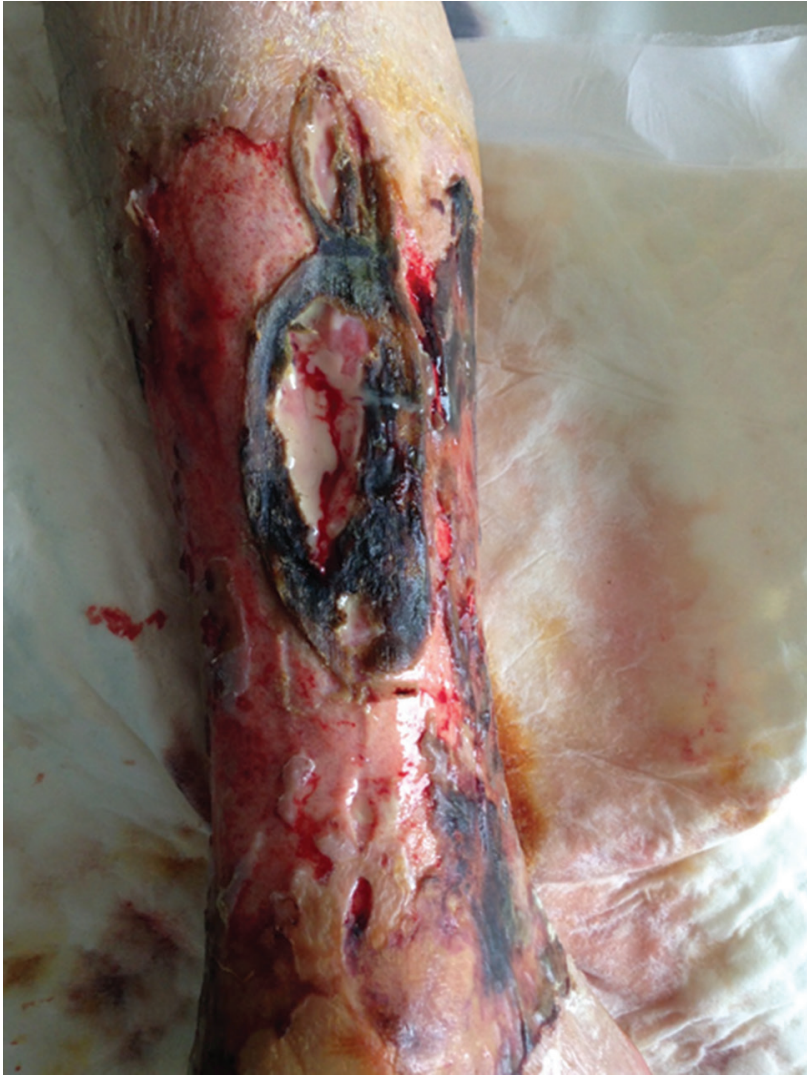

Fig 1. Wound dehiscence mixed infection.

of the grafts; the patient was in good clinical condition and was waiting for plastic surgery of calcaneus ulcer skin.

\section{Discussion}

This patient was at high risk of lower extremities amputation due to the failure of the first limb revascularisation and the presence of

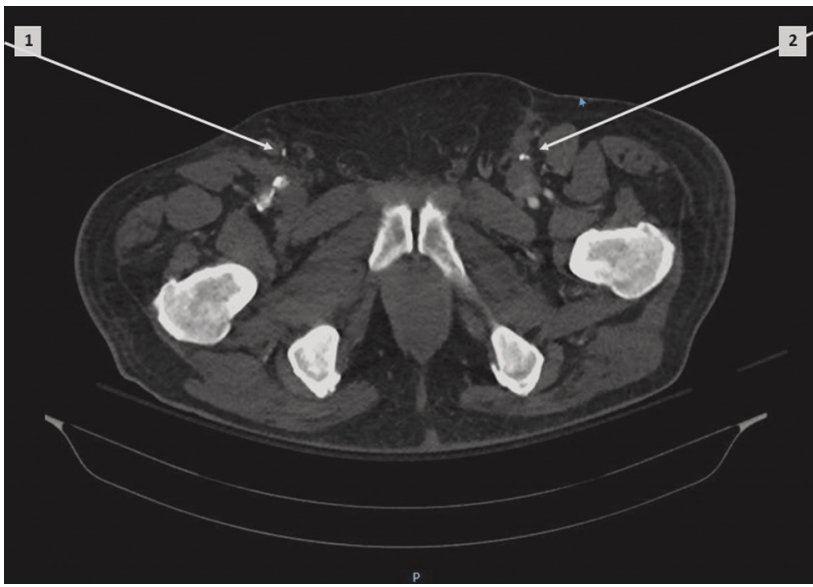

Fig 2. Axial contrast-enhanced computed tomography showing bilateral occlusion of femorotibial bypass. Right femorotibial anterior bypass (arrow 1) and left femorotibial posterior bypass (arrow 2).

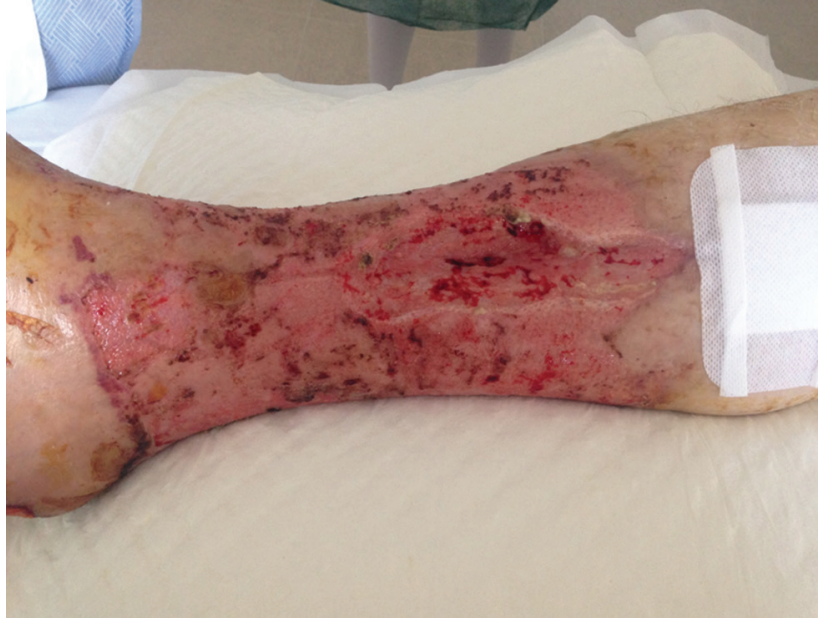

Fig 3. Wound dehiscence with methicillin-resistant Staphylococcus aureus infection.

an MRSA infection in both limb wounds, which strongly worsened the outcome. It is known that diabetic patients have an increased susceptibility to infection, Escherichia coli (25.5\%), Staphylococcus aureus (22.6\%) and Klebsiella sp (5.4\%) being the most frequent isolated from ulcer skin., ${ }^{5,6}$ The combination of diabetes with peripheral arterial disease greatly increases the risk of skin ulcers and wound infections which represent one of the major causes of hospitalisation. ${ }^{7,8}$ The risk of mortality following admission of patients with diabetic foot infection is $8.9 \%$ in 1 year. ${ }^{9}$

Probably in our patient, MRSA infection was also due to his previous hospitalisations that allowed selection and acquisition of antibiotic-resistant bacteria. ${ }^{10}$

The presence of peripheral neuropathy allows the appearance of minor injuries, such as ingrown toenails, calluses, cuts, blisters and burns, to provide a strong incentive for the development of diabetic foot infections. ${ }^{11}$

More than $10 \%$ of diabetic patients are likely to undergo extremities amputation during the first year follow-up in a multidisciplinary outpatient diabetic foot clinic. ${ }^{12}$ The risk of

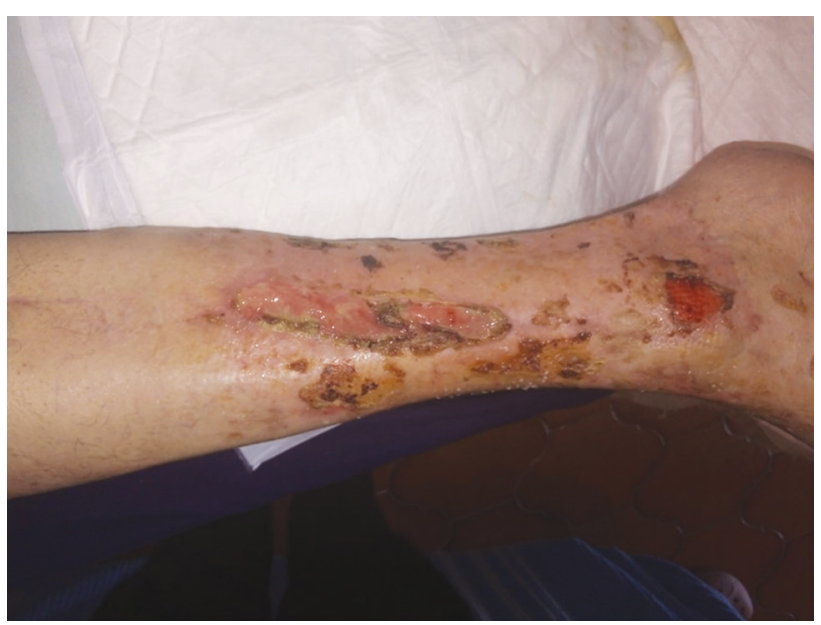

Fig 4. Healing of wound dehiscence. 
amputation may be increased when a timely and effective revascularisation is not performed and surgical debridement and antibiotic regimen are not promptly started, with a 5-year mortality up to $60 \%{ }^{13}$ Complications such as osteomyelitis, endocarditis and sepsis may also occur leading to a high mortality.

In the previous months, our patient did not get checked for glycaemia, that is one of the principal therapeutic targets to keep under control. ${ }^{14}$ However, he avoided amputation due to a prompt and effective second revascularisation together with surgical debridement, glycaemic control and antibiotic treatment of the wound infection. In particular, in accordance with the guidelines for diabetic foot infection, debridement of the wound was performed in a timely manner. ${ }^{9}$ Daptomycin and rifampicin demonstrated to be the appropriate antibiotics to treat the MRSA wound infection within 2 weeks. Then, at home, daptomycin was switched to teicoplanin, another first-line anti-MRSA agent, to ensure the therapeutic continuity and avoid relapses. ${ }^{15}$

Both physical examination (colour, temperature of the skin and palpation of peripheral pulse) and colour Doppler sonography of the lower limbs were periodically performed to warrant the good outcome of the vascular bypass over time.

Therefore, we want to emphasise the need for effective measurements of vascular risk prevention in the framework of a multidisciplinary team. Vascular risk factors advise prompt, appropriate and timely follow-up action with the aim to reduce the development of complications. Only an early recognition of peripheral arterial diseases may hasten sending the patients to reference vascular care for the necessary measures to prevent complications.

Furthermore, multidisciplinary teams can better face and more efficaciously counteract vascular and infective complications of these patients. On the other hand, diabetic patients with great ulcer size, high duration of the infection, smoking and poor glycaemic control present more frequently multidrug resistant organisms and have severe complications such as neuropathy and osteomyelitis. ${ }^{6,8}$ Therefore, these patients should be followed with the required clinical depth. Femoroperoneal bypass through lesser saphenous vein combined with wound debridement and targeted antimicrobial therapy may be efficacious in diabetics with previous femorotibial vascular occlusion and critical limb ischaemia.

Only a combined and integrated clinical, surgical and vascular team may improve their outcome and prevent severe lifethreatening complications.

\section{References}

1 Malyar NM, Freisinger E, Meyborg M et al. Amputations and mortality in in-hospital treated patients with peripheral artery disease and diabetic foot syndrome. J Diabetes Complications 2016;30:1117-22.

2 Shatnawi NJ, Al-Zoubi NA, Hawamdeh H, et al. Redefined clinical spectra of diabetic foot syndrome. Vasc Health Risk Manag 2018:14:291-8.

3 Azuma N. The diagnostic classification of critical limb ischemia. Ann Vasc Dis 2018;11:449-57.

4 Liu C, Bayer A, Cosgrove SE et al. Clinical practice guidelines by the Infectious Diseases Society of America for the treatment of methicillin-resistant Staphylococcus aureus infections in adults and children. Clin Infect Dis 2011;52:285-92.

5 Cheng NC, Tai HC, Chang SC, Chang CH, Lai HS. Necrotizing fasciitis in patients with diabetes mellitus: clinical characteristics and risk factors for mortality. BMC Infect Dis 2015;15:417.

6 Zubair M, Ahmad J. Potential risk factors and outcomes of infection with multidrug resistance among diabetic patients having ulcers: 7 years study. Diabetes Metab Syndr 2019;13:414-8.

7 Lipsky BA. Diabetic foot infection: current treatment and delaying the 'post antibiotic era'. Diabetes Metab Res Rev 2016;32(Suppl 1):246-53.

8 Lenselink E, Holloway S, Eefting D. Outcomes after foot surgery in people with a diabetic foot ulcer and a 12-month follow-up. J Wound Care 2017;26:218-27.

9 Lynar SA, Robinson CH, Boutlis CS, Commons RJ. Risk factors for mortality in patients with diabetic foot infections: a prospective cohort study. Intern Med J 2019;49:867-73.

10 Lipsky BA, Peters EJ, Senneville E et al. Expert opinion on the management of infections in the diabetic foot. Diabetes Metab Res Rev 2012;28(Suppl 1):163-78.

11 Grennan D. Diabetic foot ulcers. JAMA 2019;321:114.

12 Ferreira L, Carvalho A, Carvalho R. Short-term predictors of amputation in patients with diabetic foot ulcers. Diabetes Metab Syndr 2018:12:875-9.

13 Mavrogenis AF, Megaloikonomos PD, Antoniadou T et al. Current concepts for the evaluation and management of diabetic foot ulcers. EFORT Open Rev 2018;3:513-25.

14 Xiang J, Wang S, He Y et al. Reasonable glycemic control would help wound healing during the treatment of diabetic foot ulcers. Diabetes Ther 2019;10:95-105.

15 Choo EJ, Chambers HF. Treatment of methicillin-resistant Staphylococccus aureus bacteremia. Infect Chemother 2016:48:267-73.

Address for correspondence: Dr Enrico M Zardi, head of internistic ultrasound service and hospitalist consultant, Lower and Upper Limb Unit and Thoracic and Vascular Surgery Units, Campus Bio-Medico University, Via Álvaro del Portillo, 200, 00128 Rome, Italy.

Email: e.zardi@unicampus.it 